\title{
Reabilitação de paciente anoftálmico bilateral por meio de prótese ocular: relato de caso
}

\author{
Rehabilitación de paciente anoftálmico bilateral por medio de prótesis ocular: relato de caso \\ Rehabilitation of bilateral anoftálmic patient by eye prosthesis: case report \\ Gabriela BENTO ${ }^{1}$ \\ Letícia Rocha MARINHO' \\ Cirurgiã-Dentista, Mestranda do Programa de Pós Graduação em Ciências Odontológica, Universidade Federal de Alfenas(UNIFAL) 37.130-000 Alfenas-MG, Brasil \\ ${ }^{2}$ Cirurgiã-Dentista, Departamento de Odontologia Restauradora, Faculdade de Odontologia, Universidade Federal de Alfenas(UNIFAL) $37.130-000$ AlfenasMG, Brasil \\ ${ }^{3}$ Cirurgiã-Dentista, Mestre e Doutora em Prótese, Professora da Disciplina de Prótese Parcial Removível do Departamento de Odontologia Restauradora, \\ Faculdade de Odontologia, Universidade Federal de Alfenas (UNIFAL-MG), 37.130-000 Alfenas - MG, Brasil
}

\section{Resumo}

Introdução: A prótese ocular visa à reparação aloplástica das perdas ou deformidades do bulbo ocular, tendo por objetivos recuperar a estética facial; prevenir o colapso e a deformidade palpebral; proteger a cavidade anoftálmica contra agressões por poeira, fumaça ou poluentes; restaurar a direção da secreção lacrimal e prevenir o acúmulo deste fluido na cavidade evitando as alterações assimétricas que progressivamente se instalam. Objetivo: relatar um caso clínico de reabilitação de paciente anoftálmico bilateral por meio de prótese ocular. Material e método: Para a realização do procedimento, a cavidade anoftálmica do paciente foi moldada com alginato. Esse molde foi incluído em mufla para obtenção da esclera artificial em Resina Acrílica Ocular Termicamente Ativada (RAOTA) cor N2. A esclera obtida foi provada no paciente e recebeu os ajustes necessários. Em seguida, foi realizada a centralização da pupila, feito o platô e pintada a íris artificial, que foi colada sobre o platô. Toda a superfície da esclera recebeu caracterização e o conjunto foi recoberto com RAOTA incolor. Por fim, a prótese recebeu acabamento, polimento, e foi instalada. O paciente foi instruído quanto ao uso e higienização. Resultado: Foi observado que após a instalação da prótese, a estética facial do indivíduo foi restaurada, o suporte palpebral restabelecido e o direcionamento lacrimal corrigido, além da reinserção do indivíduo ao convívio social. Conclusão: o tratamento reabilitador atingiu seu objetivo de restaurar a estética facial e foi de grande importância para o paciente, pois o mesmo resgatou a autoestima e pôde retornar ao convívio social sem constrangimentos.

Descritores: Reabilitação; Olho Artificial; Anoftalmia.

\begin{abstract}
Introduction: The ocular prosthesis aims at the alloplastic repair of the losses or deformities of the ocular bulb, aiming to recover the facial esthetics; prevent collapse and eyelid deformity; protect the anophthalmic cavity against aggression from dust, smoke or pollutants; restore the direction of lacrimal secretion and prevent the accumulation of this fluid in the cavity avoiding the asymmetric changes that progressively install. Objective: to report a clinical case of rehabilitation of bilateral anophthalmic patient by ocular prosthesis. Material and method: To perform the procedure, the patient's anophthalmic cavity was cast with alginate. This template was included in muffle to obtain artificial sclera in thermally activated ocular acrylic resin (RAOTA) color N2. The obtained sclera was tested on the patient and received the necessary adjustments. Afterwards, the pupil was centralized, made the plateau and painted the artificial iris, which was glued on the plateau. The entire surface of the sclera was characterized and the whole was covered with colorless RAOTA. Finally, the prosthesis was finished, polished, and installed. The patient was instructed in the use and hygiene. Results: It was observed that after the installation of the prosthesis, the individual's facial aesthetics were restored, the eyelid support was restored and the corrected lacrimal targeting was performed, as well as the reinsertion of the individual into social interaction. Conclusion: the rehabilitation treatment reached its goal of restoring the facial aesthetics and was of great importance for the patient, since it rescued the self-esteem and was able to return to the social life without constraints.

Descriptors: Rehabilitation; Artificial Eye; Anophthalmia.
\end{abstract}

\section{Resumen}

Introducción: La prótesis ocular se refiere a la reparación aloplástica de las pérdidas o deformidades del bulbo ocular, teniendo como objetivo recuperar la estética facial; prevenir el colapso y la deformidad palpebral; proteger la cavidad anoftálmica contra agresiones por polvo, humo o contaminantes; restaurar la dirección de la secreción lagrimal y prevenir la acumulación de este fluido en la cavidad evitando los cambios asimétricos que progresivamente se instalan. Objetivo: relatar un caso clínico de rehabilitación de paciente anoftálmico bilateral por medio de prótesis oculares. Material y método: Para la realización del procedimiento, la cavidad anoftálmica del paciente fue moldeada con alginato. Este molde fue incluido en mufla para la obtención de la esclera artificial en Resina Acrílica Ocular Termalmente Activada (RAOTA) color N2. La esclera obtenida fue probada en el paciente y recibió los ajustes necesarios. A continuación, se realizó la centralización de la pupila, hecha la meseta y pintada el iris artificial, que fue pegada sobre la meseta. Toda la superficie de la esclera recibió caracterización y el conjunto fue recubierto con RAOTA incoloro. Por último, la prótesis recibió el acabado, pulido, y se instaló. El paciente fue instruido en cuanto al uso e higienización. Resultado: Se observó que después de la instalación de la prótesis, la estética facial del individuo fue restaurada, el soporte palpebral restablecido y el direccionamiento lagrimal corregido, además de la reinserción del individuo a la convivencia social. Conclusión: el tratamiento rehabilitador alcanzó su objetivo de restaurar la estética facial y fue de gran importancia para el paciente, pues el mismo rescató la autoestima y pudo regresar a la convivencia social sin limitaciones.

Descriptores: Rehabilitación; Ojo artificial; Anoftalmía.

\section{INTRODUÇÃO}

A reabilitação por meio de prótese ocular é o tratamento de escolha quando não é possível manter o globo ocular, totalmente ou em parte. Essa prótese recupera a estética e a autoestima do paciente anoftálmico, mantendo a cavidade anoftálmica preenchida e possibilitando que o fluído lacrimal seja direcionado e não fique acumulado na cavidade. A prótese possui também a função de prevenir a deformação das pálpebras, proteção da mucosa interna sensível e manutenção do tônus muscular, evitando assim a assimetria facial ${ }^{1}$.

De acordo com o método utilizado para sua confecção as próteses oculares podem ser classificadas em duas categorias: Próteses PréFabricadas: são próteses de estoque, disponíveis em vários tamanhos e cores, adaptadas à cavidade do 
paciente. Estas próteses são de rápida aquisição, porém, nem sempre conseguem restabelecer a estética e a movimentação de maneira satisfatória; e Próteses Individualizadas: confeccionadas a partir de um molde da cavidade anoftálmica, que dará origem a uma prótese perfeitamente adaptada as estruturas do paciente, restaurando a estética perdida e promovendo movimentação adequada da prótese, conforto e proteção dos tecidos adjacentes ${ }^{2}$.

\section{CASO CLÍNICO}

Paciente, sexo masculino, 29 anos de idade, melanoderma, transcritor, apresentou-se à clínica de Odontologia da UNIFAL-MG queixando-se de necessidade protética. $\mathrm{Na}$ anamnese, relatou ter microftalmia e glaucoma congênito; aos 12 anos de idade passou por cirurgia para estabilizar o glaucoma e a última cirurgia foi há 13 anos para correção da atrofia ocular; relatou nunca ter utilizado próteses oculares (Figura 1).

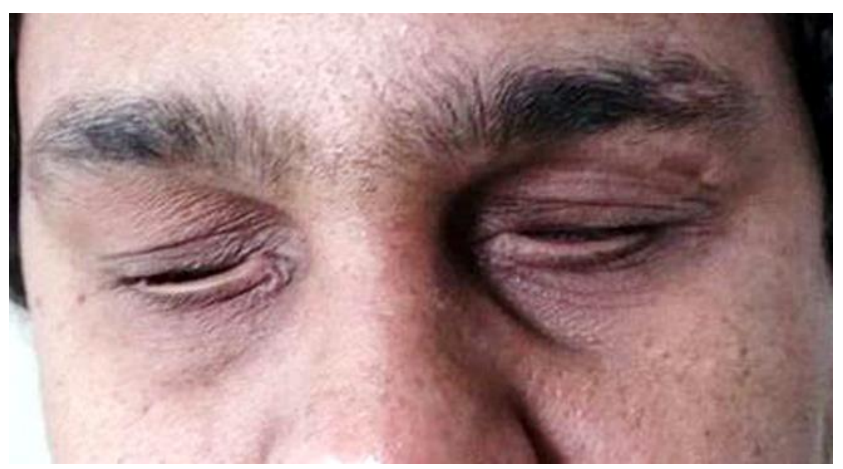

Figura 1: Aspecto inicial do paciente, sem próteses oculares.

Inicialmente foram confeccionadas moldeiras em resina acrílica quimicamente ativada (Figura 2). Essas moldeiras receberam acabamento e foram provadas nas cavidades anoftálmicas do paciente, foram selecionadas aquelas que promoviam melhor afastamento palpebral, o que facilitaria a moldagem. Em seguida, foi realizada uma perfuração central nas moldeiras a fim de se promover a adaptação das mesmas a uma seringa descartável, e diversas perfurações menores para favorecer a retenção do material de moldagem à moldeira.

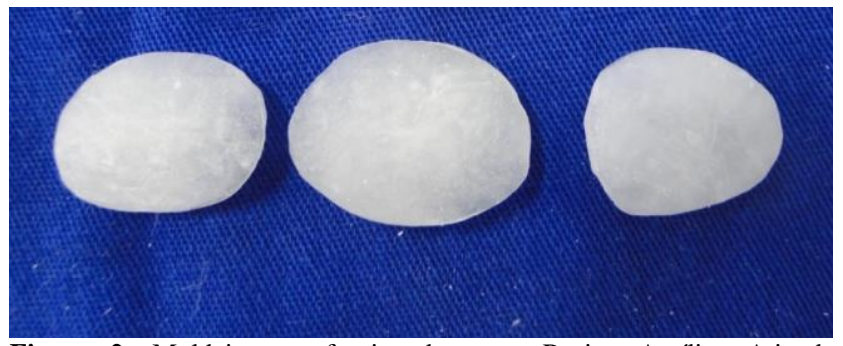

Figura 2: Moldeiras confeccionadas com Resina Acrílica Ativada Quimicamente (RAAQ).

A partir daí, teve início a moldagem das cavidades (Figura 3). O material escolhido foi o alginato, que foi proporcionado, manipulado $\mathrm{e}$ inserido na seringa descartável já com a moldeira acoplada em sua extremidade. As moldeiras foram posicionadas entre as pálpebras do paciente e o alginato foi injetado lentamente. Durante esse procedimento, foi solicitado ao paciente que realizasse movimentos com a musculatura orbicular para a obtenção de uma cópia fiel de todos os detalhes das cavidades. Após a geleificação do material de moldagem o molde foi removido do interior das cavidades do paciente e avaliado quanto sua qualidade.

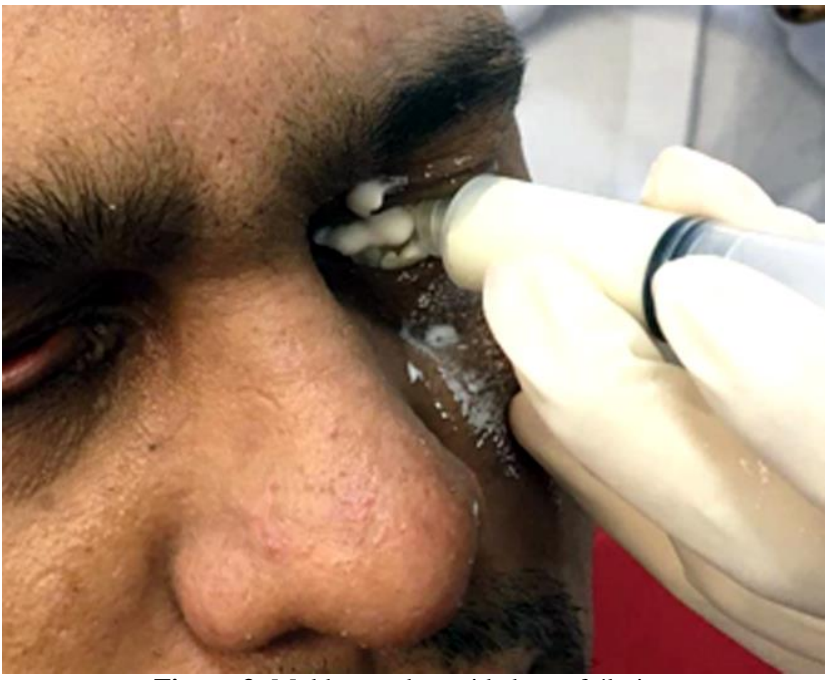

Figura 3: Moldagem da cavidade anoftálmica.

Em seguida, foram confeccionados os moldes para prensagem da Resina Acrílica Ocular Termicamente Ativada (RAOTA) para obtenção das escleras artificiais (Figura 4), para isso, a silicona de condensação de consistência densa foi manipulada de acordo com as instruções do fabricante, e também foi utilizada para recobrir uma das faces dos moldes de alginato obtidos. Polimerizada a silicona, o conjunto molde-moldeira foi incluído em mufla preenchida com gesso comum (Figura 4-A). Decorrido o tempo de cristalização do gesso, a outra face dos moldes foi recoberta com silicona, como descrito previamente (Figura 4-B). Após a polimerização do material, a superfície do gesso exposta foi vaselinada, a contramufla posicionada, preenchida com gesso e levada para uma prensa de bancada. Decorrido o tempo de cristalização do gesso, a mufla foi removida da prensa, aberta e o conjunto molde-moldeira foi removido, criando, assim, um molde para prensagem da Resina Acrílica Ocular Termicamente Ativada (Figura 4-C).

A etapa seguinte consistiu na obtenção das escleras artificiais. Para isso, a RAOTA da cor N2 foi manipulada, inserida no molde de silicone dentro da mufla e polimerizada seguindo as instruções do fabricante.

Após o resfriamento, a mufla foi aberta e as escleras artificiais removidas. Foi realizado o processo de acabamento com maxicut e polimento com escova e pedra pomes seguido por e disco de feltro e branco de Espanha. Em seguida, as escleras já 
polidas foram mergulhadas em água por 24 horas para eliminação de monômero residual. Na sessão seguinte, as escleras foram provadas nas cavidades anaftálmicas do paciente. Durante essa etapa foi avaliado o preenchimento das cavidades, o contorno das pálpebras, as movimentações das escleras e o selamento palpebral.

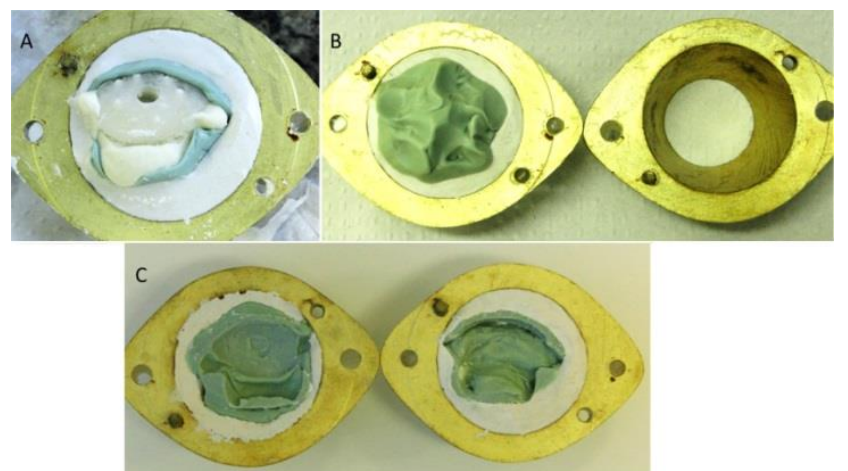

Figura 4: Inclusão do molde de alginato para obtenção de molde para prensagem de RAOTA para confecção da esclera artificial. A- Inclusão do conjunto molde-moldeira revestido por silicone em mufla. B- Recobrimento do conjunto com silicone para preenchimento da segunda parte da mufla. C- Mufla aberta após a remoção do conjunto, mostrando o molde para prensagem da RAOTA.

Realizados os ajustes necessários, as pupilas foram centralizadas, e foi feita redução da curvatura anterior superficial das escleras em $1 \mathrm{~mm}$ através do platô. As íris artificiais foram pintadas sobre papel cartão na cor preta, usando tinta a óleo misturada a secante de cobalto. Em seguida, foi realizada a caracterização das escleras através da adição de pigmentos e fios do kit de caracterização Peter Thomas. Após a secagem dos pigmentos, as íris artificiais foram coladas sobre os platôs com cola branca escolar (Figura 5).

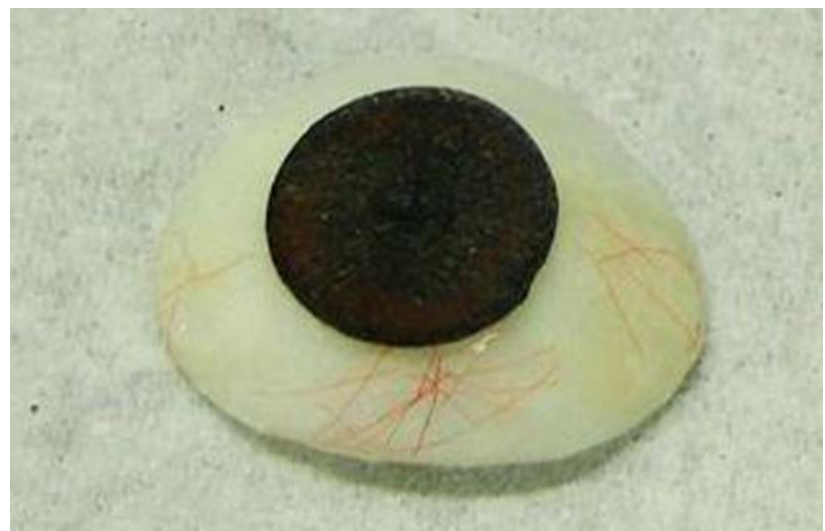

Figura 5: Esclera caracterizada e íris artificial colada sobre o platô.

Decorrida a fase de pintura, uma pequena porção de RAOTA incolor foi manipulada e inserida na mufla do lado correspondente ao que, então, recobriria a pintura e a caracterização realizadas previamente sobre as escleras. As escleras caracterizadas foram posicionadas sobre essa resina, a mufla foi fechada, posicionada em prensa de bancada com pressão de 1,25 toneladas por 10 minutos, os excessos de resina foram recortados e a mufla foi transferida para uma prensa de mão, a fim de se realizar a polimerização térmica da RAOTA incolor.

A RAOTA incolor foi polimerizada seguindo as orientações do fabricante. Após o resfriamento, a mufla foi aberta, as próteses oculares removidas e submetidas a acabamento e polimento. As próteses foram instaladas (Figura 6), e o paciente foi instruído quanto ao uso e higienização, além de ter sido alertado a respeito da necessidade de polimentos periódicos para correção de possíveis riscos advindo do processo de higienização e/ou quedas da prótese e da necessidade de substituição quando ocorrer desadaptação. No momento da instalação, o paciente demonstrou muita satisfação com o tratamento realizado, especialmente no que dizia respeito à adaptação das próteses, e sua acompanhante relatou estar satisfeita com a estética obtida.

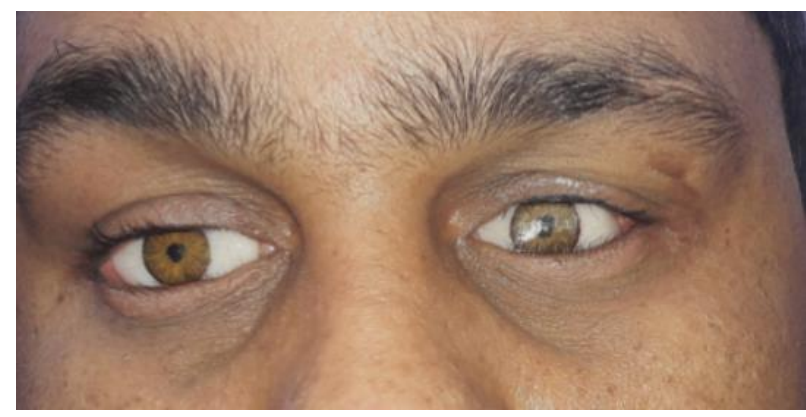

Figura 6: Próteses oculares instaladas.

DISCUSSÃO

Para reabilitação do paciente anoftálmico diferentes tipos de próteses são propostas; a opção por cada uma delas dependerá das características da cavidade, presença ou não de remanescente do globo e relação com a etapa cirúrgica ${ }^{3}$. De acordo com as características do caso relatado, e pelo fato de o paciente nunca ter utilizado nenhum tipo de prótese anteriormente optou-se pela execução de uma prótese ocular restauradora individualizada, já que essa oferece um resultado estético e funcional superior à prótese de estoque. A prótese de estoque é muito procurada pelos pacientes por ser de rápida aquisição, porém não oferece a mesma qualidade da individualizada, a mobilidade é limitada e pode promover alterações prejudiciais à cavidade anoftálmica ${ }^{4,5}$.

Para a moldagem da cavidade anoftálmica, diferentes tipos de materiais odontológicos podem ser utilizados, tais como ceras, hidrocoloide irreversível e materiais elastoméricos ${ }^{6}$. No presente caso foi utilizado o hidrocoloide irreversível por possuir fácil escoamento, permitindo um preenchimento total da cavidade.

Com relação às técnicas de moldagem a literatura cita impressão direta, impressão externa, impressão com moldeira de estoque, impressão com moldeira ocular personalizada, com prótese ocular de 
estoque, com prótese ocular modificada e confecção de esclera em cera. Algumas técnicas dependem de o paciente já ser usuário de prótese ocular e outras da conformação da cavidade anoftálmica. A eficácia e escolha de cada método dependem da experiência do profissional e dos materiais disponíveis ${ }^{6}$. No presente relato clínico, foi utilizada a técnica descrita por Maia et al. ${ }^{4}$, Mathews et al. ${ }^{7}$ e Bartlett e Moore ${ }^{8}$ na qual a moldagem é realizada através da injeção do material com seringa plástica e com moldeira individual de resina acrílica, perfurada com uma adaptação de um conduto central para acoplar a seringa, confeccionada em vários tamanhos para selecionar a que melhor se encaixa.

A partir da moldagem, as escleras foram confeccionadas. A resina acrílica é o material de escolha para a sua confecção, devido as suas qualidades como: baixo custo, fácil manuseio, adequados valores de resistência, durabilidade, facilidade de limpeza, retenção mecânica confiável e biocompatibilidade ${ }^{6,9}$. Como o paciente possuía microftalmia bilateral e nunca havia utilizado prótese ocular antes, para a confecção da íris, no presente caso, foram observados aspectos físicos do paciente, tais como cor da pele e idade. Em seguida, iniciou-se a pintura com tinta a óleo em disco de papel cartão preto, seguida da pupila, halo peripupilar e halo externo preconizando as técnicas descritas por Goiato et al. ${ }^{6}$ e Helland ${ }^{10}$.

Outra opção viável para a reprodução de íris artificiais é a utilização da imagem digital. A utilização da imagem digitalizada da íris remanescente obtida por fotografia digital e impressa em papel através de impressão com jato de tinta apresenta além de resultados estéticos bastante favoráveis, algumas outras vantagens sobre a pintura tradicional, como: técnica simples, tempo de tratamento menor, além de requerer habilidades artísticas mínimas. Exige, porém a utilização de equipamento especial, além da necessidade de recobrir a imagem com um adesivo importado de difícil obtençãa ${ }^{6,11}$. Porém, no presente relato não foi possível fazer a reprodução fotográfica nem tampouco observar a íris sadia porque a perda do paciente era bilateral.

Finalizada a pintura da íris foi realizada a caracterização dos vasos sanguíneos através de fibras de seda vermelha, a esclera deve ser recoberta por uma camada de resina acrílica incolor que pode variar de 1 a $3,5 \mathrm{~mm}$, permitindo, pela sua translucidez, a perfeita visualização da íris artificial, assim como descrito por Goiato et al. ${ }^{6}$.

A prótese ocular do caso em questão recebeu essa cobertura e, por fim, acabamento e polimento adequado. Em seguida a prótese foi instalada observando-se a adaptação, a mobilidade a estabilidade e a estética. $O$ paciente recebeu instruções de uso e dos cuidados diários de higienização. Foram recomendado retornos periódicos para avaliação e acompanhamento da necessidade de troca da prótese ${ }^{9}$.

\section{REFERÊNCIAS}

1. Raiazada K, Rani D. Ocular prosthesis. $\mathrm{J} \mathrm{Br}$ Contact Lens Assoc. 2007; 30(3):152-62.

2. Maghami MH, Sodagar AM, Lashay A, RiaziEsfahani M. Visual prostheses: The enabling technology to give sight to the blind. J Ophthalmic Vis Res. 2014; 9(4):494-505.

3. Rezende JRV. Fundamentos da prótese bucomaxilo-facial. São Paulo: Sarvier; 1997.

4. Maia FAS, Dias RB, Rezende JRV. Estudo comparativo das técnicas de moldagem da cavidade anoftálmica visando a confecção da prótese ocular. Rev Odontol Univ São Paulo. 1997; 11(suppl 1):85-90.

5. Kumar CH S, Sajjan CS. Prosthetic management of an ocular defect. Contemp Clin Dent. 2010; 1(3):201-3.

6. Goiato MC, Bannwart LC, Haddad MF, dos Santos DM, Pesqueira AA, Miyahara GI. Fabrication techniques for ocular prostheses--an overview. Orbit. 2014; 33(3):229-33.

7. Mathews MF, Smith RM, Sutton AJ, Hudson R. The ocular impression: A review of the literature and presentations of an alternative technique. $\mathrm{J}$ Prosthodont. 2000; 9(4):210-16.

8. Bartlett SO, Moore DJ. Ocular prosthesis: A physiologic system. J Prosthet Dent. 1973; 29(4):450-59.

9. Emídio TCS, Dutilh JDAM, Moro MC, Dutilh CM. Reabilitação com prótese ocular individualizada em pacientes jovens: relato de casos clínicos. Int J Dent. 2011; 10(3):190-94.

10. Helland M. Fabrication of ocular prostheses. In: Beumer J, Curtis TA, Firtell DN (eds). Maxillofacial rehabilitation: prosthodontic and surgical considerations. St Louis: CV Mosby; 1979. pp. 352-62.

11. Artopoulou II, Montgomery PC, Wesley PJ, Lemon JC. Digital imaging in the fabrication of ocular prostheses. J Prosthet Dent. 2006; 95(4):327-30.

\section{CONFLITO DE INTERESSES}

Os autores declaram não haver conflitos de interesse.

AUTOR PARA CORRESPONDENCIA

Marcela Fillié Haddad

marcela.haddad@unifal-mg.edu.br

Submetido em 12/04/2018

Aceito em 27/06/2018 